\title{
电气工程自动化的智能化技术应用与研究
}

\author{
李国令 \\ 亚龙智能装备集团股份有限公司，浙江 温州 325105
}

\begin{abstract}
[摘要]信息科技不断发展, 智能化技术应用而生并且发挥越来越重要作用。在电气工程中, 自动化技术的应用已经推动电气 工程得到较大发展, 但是该技术在飞速发展过程中也逐渐暴露出一些不足, 比如工作效率低、工序流程繁琐等。智能化技术 应用能够有效解决这些问题。在电气工程中应用智能化技术可以将企业生产效率和经济效益大大提升, 能够优化电气设备控 制效率和准确性。为此，需要重点探讨智能化技术在电气工程中的应用，充分发挥出智能化技术优势。
\end{abstract} [关键词]电气工程; 自动化; 智能化技术

DOI: $10.33142 / \mathrm{aem} . v 2 \mathrm{i} 11.3202$ 中图分类号：TM76 文献标识码：A

\section{Application and Research of Intelligent Technology in Electrical Engineering Automation}

LI Guoling

Yalong Intelligent Equipment Group Co., Ltd., Wenzhou, Zhejiang, 325105, China

\begin{abstract}
With the continuous development of information technology, intelligent technology is emerging and playing an increasingly important role. In electrical engineering, the application of automation technology has promoted the development of electrical engineering, but in the process of rapid development, the technology also gradually exposed some shortcomings, such as low efficiency, cumbersome process and so on. The application of intelligent technology can effectively solve these problems. The application of intelligent technology in electrical engineering can greatly improve the production efficiency and economic benefits of enterprises and optimize the control efficiency and accuracy of electrical equipment. Therefore, we need to focus on the application of intelligent technology in electrical engineering and give full play to the advantages of intelligent technology.
\end{abstract}

Keywords: electrical engineering; automation; intelligent technology

\section{1 智能化技术的有关概念与其各项优势}

\section{1 有关概念}

高水平计算机技术的重要组成部分之一就是智能技术。智能化技术主要是利用智能技术对人脑进行模拟, 让机器 能够仿照人脑开展独立思考, 从而更加快捷、高效地收集、加工相关资料。智能化技术在语言学、生物学、控制学等方 面都有所涉及, 其综合性和技术性较强, 同时人们的生活工作方式也随着智能化技术的发展在不断改善。和传统常规技 术相比, 智能化技术能够在电气工程自动化中全部动态化监管控制对象, 能够自动将没有关联的因素排除掉。和传统机 电工程安装相比, 智能化机电设备需要涉及到更多的内容, 有着更高的安装要求。智能化工程包含诸多的内容, 比如最 为典型的应用之处为弱电智能化系统。弱电智能化系统的应用需要涉及到诸多子系统, 在集成系统之前首先需要深入分 析各个应用子系统, 根据不同子系统的特点集成各个内容, 统一规划各个系统, 确保各个系统都能够充分发挥其价值。

\section{2 智能化技术对于控制模型构建所具有的优势}

电气控制对象本身的复杂性导致传统自动化控制中的精准度难以达到现如今市场需求, 同时受到各类不确定因素 的影响, 在电气工程控制中容易出现预测偏差等不同程度的问题。智能化技术可以直接省略了设计被控制对象模型的 过程, 避免了不可控因素导致的精准度降低问题，可以将电气工程控制的精准度大大提升。

\section{3 智能化技术本身的一致性优势}

在电气工程自动化中应用智能化技术能够将智能化技术的一致性价值发挥出来。在智能化技术应用于电气自动化 控制之后可以准确地评估任何一项输入的数据, 可以快速评估一些不常用的数据, 将评估的效率和质量全面提高。此 外, 控制效果受到不同控制对象变更性的差异影响导致出现一定的不同。当前即使是应用了智能化技术也很难高效地 解决这一问题。为此, 未来发展中, 需要加强优化智能化技术在电气工程自动化控制中的应用, 进一步发展智能化技术。

\section{4 智能化技术本身的控制精度优势}

传统的电气工程自动化控制无需对被控制对象进行建模处理, 各种不确定因素都可能影响建模的过程。只有加强 把控不确定因素才能达到更加精准地控制对象的目的, 也正是因此智能化技术开始应用于电气工程自动化中, 将建模 这一过程省略从而达到不确定因素干扰的排除效果, 将智能化控制的精准度提升。 


\section{5 智能化技术本身的控制水平优势}

在电气工程自动化中应用智能化技术可以将电气系统的整体控制水平提升, 能够达到程序控制和数据控制能力的 优化。同时, 在电气工程自动化中应用智能化技术还可以及时发现其中存在的问题并且通过平台发出警示提示工作人 员及时处理问题, 避免问题扩大产生更加严重的后果, 可以达到控制故障发展的效果, 能够避免发生严重的突发事件, 实现了电气系统运行质量、稳定性提升的效果。

\section{6 智能化技术本身的综合性控制优势}

智能化技术在电气工程自动化中的应用必然会存在一些不足之处, 在发展过程中我们需要不断改进创新, 优化智 能化技术的应用, 实现更加精准、高效地控制电气工程。为此, 工作人员需要加强关注智能化技术的不足和创新, 积 极采用动静结合的方式推动电气工程的发展，充分发挥智能化技术的优势。

\section{2 智能化技术在电气工程自动化控制中的具体应用}

\section{1 优化产品设计}

在建设电气自动化系统过程中重要的基础就是电气设备, 电气设备直接关系着整个电气系统的运行。在设计过程 过程中, 传统电气设备设计需要耗费大量的时间, 所以容易导致出现时间过长、误差增大的现象, 同时也不利于提高 电气系统的运行效率。电气系统和设备设计中对专业技术有着较高的要求, 相关工作人员不但要有专业的技术能力最 好还要具备丰富的工作经验, 只有这样才能对电气产品设计和运行中出现的问题及时发现并且进行深入地研究, 才能 为设备创造有利的运行环境, 才能提高电气设备的运行可靠性。可以采用数据算法的方式实现优化电气设备管理工作 的效果。不过在使用传统数据算法过程中可能会导致整体设计过程中有着较大的工作量, 复杂程度也较高, 此时应用智 能化技术方式可以将设备运行过程中出现的不同类型的损耗问题进一步降低。在使用电气系统过程中利用智能化技术可 以实现系统自我评价和工作人员评价结合的方式, 为工作人员提供数据参考, 节省工作人员工作量, 提高电气设计效率。

遗产算法是当前电气工程产品设计中常用的一种方式。工作人员在具体开展产品设计过程中受到工作经验和设计 要求方面的影响可能会出现电气设备设计差异性的问题。随着社会发展、电气生产水平的提升、生产需求多样化发展, 各个行业在电气控制方面的要求也越来越高。在人工涉及电气系统过程中可能会存在较多的缺陷, 而应用智能化后可 以将设计准确度和效率提升, 有助于减少设计缺陷。利用智能化技术科学指导电气设计, 可以将分析和检测系统的效 率提高, 有助于达到管理精细化的目的, 有助于对各个环节故障问题进行监测, 实现电气系统整体运行效果的优化。 作为以自然界进化规律为基础的算法, 遗产算法能够分析确定最优化系统设计方案, 可以对每个环节进行精细化设计, 从而预测和计算系统故障, 并且对比不同类型的设计方案, 分析各个方案的优缺点, 帮助工作人员科学地确定设计方案。

\section{2 故障的监控和处理}

在设计电气自动化系统中难免会遇到设备故障或者人为事故。智能化技术可以有效处理这两种情况，比如在发动机运行 中能够有效处理相关故障。传统处理带故障运行变压器主要是进行气体样本的收集然后分离和分析气体, 最后根据分析结果 对机器故障可能原因进行判定。采用这种处理方式需要耗费大量的人员和时间, 并且处理效率较低, 还存在较大的误诊的可 能性。智能化技术相比于传统故障处理法昂视更加优越, 如果发生故障系统可以纵向对比故障, 对故障产生原因进行自动分 析, 并且匹配出相关的处理方案。通过在电气故障管理中应用智能化技术可以将问题处理效率和准确性大大提升。

\section{3 智能控制}

将智能化技术应用于电气自动化系统中能够实现智能化操作控制一些设备仪器。比如通过在各种电气设备上应用 智能化技术可以高效率地完成电气设备的控制管理。当前模糊控制、神经网络控制都是常用的电气设备控制方式。在 日常控制和操作控制中和传统的人工操作电气系统的控制方式相比, 智能控制能够将操作误差降低, 有助于提高控制 的准确性和高效性, 有助于节省劳动力, 有利于推动电气自动化控制系统的发展。将智能化技术引入到电气工程中能 够综合性控制电气系统, 实现自动化管理模式, 从而保证电气系统能够稳定地运转。同时, 相关工作者应当加强对智 能化控制系统的重视也研究, 在管理和操作过程中将精准度不断提高, 加强总结经验教训, 积极改进创新, 搭建神经 网络控制系统，模拟人类大脑思维运行方式，模拟人脑进行指令控制，从而将电气控制的智能化、安全性和可靠性提高。

\section{3 结语}

智能化技术在电气工程及其自动化控制中具有极为显著的优势, 对提高电气系统的运行能力以及自动化控制效果 有着积极的作用。整体而言, 智能化技术在电气工程领域有着较为可观的发展前景, 除了需要立足于实际生产的真实 需求, 也应在人才培养和技术创新等方面加大力度, 为电气工程及其自动化技术的现代化发展提供更强劲的动力。

\section{[参考文献]}

[1]井萌,古东明.浅析智能化技术在电气工程自动化控制中的应用 [J]. 世界有色金属, 2018(20): 263-264.

[2]赵妮妮. 浅谈电气工程及其自动化的智能化技术应用 [J]. 信息记录材料, 2018, 19 (12): 90-91.

[3] 梅强. 智能化技术在电气工程自动化控制中的应用探讨 $[\mathrm{J}]$. 计算机产品与流通, 2018 (11): 67 .

作者简介: 李国令 (1976.9-) 男, 职称高级技师, 毕业学校电子科技大学, 工作单位亚龙智能装备集团股份有限公司。 